\title{
Treatment of brain metastases
}

\section{TRATAMENTO DAS METÁSTASES ENCEFÁLICAS}

\author{
Authorship: Sociedade Brasileira de Neurocirurgia \\ Participants: Frederico de Melo Tavares de Lima $^{1}$, Ana Lucia Mello de Carvalho ${ }^{1}$, Joacil Carlos da Silva Jr. ${ }^{1}$, \\ Robson Amorim ${ }^{1}$, Marcos Dellaretti ${ }^{1}$, Leonardo Augusto Wendling-Henriques ${ }^{1}$, Marcos Maldaun ${ }^{1}$, \\ Daniel Kirchhoff ${ }^{1}$, Ricardo Vieira Botelho ${ }^{1}$

\section{Conflict of interest: none}

${ }^{1}$ Sociedade Brasileira de Neurocirurgia

The Guidelines Project, an initiative of the Brazilian Medical Association, aims to combine information from the medical field in order to standardize procedures to assist the reasoning and decision-making of doctors.

The information provided through this project must be assessed and criticized by the physician responsible for the conduct that will be adopted, depending on the conditions and the clinical status of each patient.

\section{INTRODUCTION}

Metastases are the most common cancers that affect the intracranial space. They have an incidence of 6 to 10 times that of primary brain tumors. The estimate is that they occur in 20 to $40 \%$ of all malignant neoplasms affecting the human body, being symptomatic in 10 to $20 \%$ of these patients. Prevalence is growing due to aging of the population, greater access to neuroimaging techniques and increased survival of patients with malignancies. Brain metastases (BM) occur with equal frequency in men and women, and have the same effect in all races. Any form of cancer can promote secondary spread in the brain. Primary tumors that contribute the most to BMs are, in descending order: lung, breast, melanoma, kidney and colorectal carcinoma. However, in over $15 \%$ of cases the primary site is unknown. Brain metastases are most often diagnosed in patients with a known malignancy (metachronous presentation). Less frequently, the lesions are diagnosed simultaneously with the primary tumor (synchronous presentation), or even before the discovery of the primary disease. About $85 \%$ of BMs are located in the supratentorial compartment; 10 to $15 \%$ are in the infratentorial space; and 1 to $3 \%$, in the brainstem. The most common symptoms in BMs are: headache, seizures, behavioral changes, hemiparesis, vomiting, dysphasia, and consciousness disorders. Most metastases spread in the brain through the blood but, less often, through the venous plexus. Without treatment, the median survival is less than 2 months.

In Brazil, based on the estimate for 2012 by the National Cancer Institute, and considering the probable 90,300 new cases of breast, lung and colon cancer, and melanoma, it is possible to estimate the occurrence of 13,545 new cases of BM per year. That number places BMs as the fourth most common form of cancer in Brazil, regardless of gender. Furthermore, it is an incidence roughly three times higher than all tumors of the central nervous system taken together.

Treatments include surgery, radiosurgery and radiotherapy, and in some cases chemotherapy. Treatment can include one method alone, or a combination of therapies.

\section{Овjective}

The aim is to clarify the effect of surgery, radiotherapy and radiosurgery in patients with brain metastases.

\section{Grade of Recommendation AND Strength of EVIDENCE}

The grade of recommendation and strength of evidence were defined in accordance with the Brazilian Medical Association, as displayed in the Table 1.

\section{TYPES OF STUDY}

The primary study for definition of this guideline is the randomized clinical trial. Clinical studies of lower methodological quality were used in the absence of the first.

\section{1) Clinical question}

What is the benefit of surgery in the treatment of brain metastases compared to radiotherapy?

Description of the evidence collection method

The search strategy was conducted in the MedLine (PubMed) database to identify articles published from 1964 to 2013. The objective was to identify studies comparing patients treated with surgery and radiotherapy with those undergoing radiation therapy alone. 
TABLE 1 Grade of recommendation and strenght of evidence.

\begin{tabular}{lll} 
Recommendation & Evidence & Type of article \\
\hline A & 1A & Systematic review (with homogeneity) of randomized controlled trials \\
\hline & 1B & Randomized controlled trials with narrow confidence interval \\
\hline 1C & All or none therapy outcomes \\
\hline B & Systematic reviews (with homogeneity) of cohort studies \\
\cline { 2 - 3 } & 2B & Cohort studies (including low-quality RCTs) \\
\hline $3 \mathrm{~A}$ & Outcomes research, ecological studies \\
\hline $3 \mathrm{~B}$ & Systematic review (with homogeneity) of case-control studies \\
\hline C & Case-control study \\
\hline D & Case-reports (and poor quality cohort and case-control studies) \\
\hline
\end{tabular}

The following terms were searched as "MeSH" and words in the text:

- 1\# (((“brain”[MeSH Terms] OR “brain”[All Fields]) AND ("neoplasm metastasis"[MeSH Terms] OR ("neoplasm"[All Fields] AND "metastasis"[All Fields]) OR "neoplasm metastasis"[All Fields] OR "metastasis"[All Fields]))

- 2\# "brain neoplasms/secondary"[MeSH Terms] OR "supratentorial neoplasms/secondary"[MeSH Terms] AND (("surgery"[Subheading] OR "surgery"[All Fields] OR "surgical procedures, operative"[MeSH Terms] OR ("surgical”[All Fields] AND "procedures"[All Fields] AND "operative"[All Fields]) OR "operative surgical procedures"[All Fields] OR "surgery”[All Fields] AND "surgery"[All Fields]) OR "microsurgery”[MeSH Terms]

Related articles were searched from the citations in the primary texts.

- Inclusion criteria: Only randomized controlled trials were evaluated, including those published in English, Spanish and Portuguese.

- Clinical outcomes included were: Functional independence, survival, tumor control, cost-effectiveness, quality of life, cognitive decline and other adverse effects.

- Combined results of searches: 7,963 articles were initially retrieved. 366 studies were considered clinical trials, of which only three were randomized and selected for critical evaluation of the strength of evidence. The remaining non-randomized were excluded.

\section{Methodological quality analysis}

The three works were classified according to the Jadad scale as $\geq 3$. Considering the size of the samples in the three studies, the one by Patchel ${ }^{1}$ (1B) had a strength of 91.93\%, while the studies by Vecht ${ }^{2}$ (1B) and $\operatorname{Mintch}^{3}$ (1B) had the same strength, $16.96 \%$.
The study by Patchell ${ }^{1}$ (1B) (1990) was randomized at a single center, and included 48 patients to compare surgery, followed by radiotherapy and biopsy plus radiotherapy. Patients had mean age of 60 years; the mean Karnofsky score was 90, confirming the good functional status of patients. Randomization was done by computer but the assessment of outcomes was not made by independent observers or blinded to the treatment. The outcomes studied were survival, functional independence, tumor size progression, time to recurrence and cause of death. Statistical analysis was performed with survival study (Kaplan-Meier and log rank test).

The study by Vecht ${ }^{2}$ (1B) (1993) was a Dutch multicenter randomized clinical trial of surgical resection, followed by whole brain radiotherapy versus radiotherapy alone. 63 patients were randomized (mean age 60 years). Randomization was done in blocks, controlled by call center, but outcome assessors were not blinded to treatment. Assessment measures included survival, functionally independent survival and cause of death.

The third ${ }^{3}$ (1B) (Mintz, 1996) was a Canadian multicenter study, comparing surgical resection followed by whole brain radiotherapy versus whole brain radiotherapy alone. The authors randomized 84 patients (mean age 59 years). Randomization was based on call center after stratification for prognostic factors. Outcome assessors were not blinded for treatment type. Outcomes included survival (percentage), cause of death, functional status (Karnofsky) and quality of life (using the Spitzer scale) and surgical complications after 30 days.

\section{Outcome data extraction}

Three types of outcomes were extracted and evaluated as clusters in the three randomized trials: Survival time, percentage of lesion recurrence, and time of functional independence. 


\section{Survival time}

The study by Patchel (1B) showed 40 weeks of survival in the surgical group and 15 weeks in the group undergoing radiotherapy $(\mathrm{RT})(\mathrm{p}<0.01)$, favoring surgical treatment.

Vecht (1B) found a longer survival (10 months) in the surgical group compared with the RT group ( 6 months) $(\mathrm{p}=0.04)$.

In the study by Mintz (1B), there was no difference regarding survival: 5.62 months in the surgical group, 6.28 months in the RT group ( $\mathrm{p}=0.24)$.

The analysis of randomized trials based on survival curves has several limitations. ${ }^{4,5}$ The survival analysis did not show a statistically significant difference between the two treatments $(\mathrm{HR}=0.72,95 \mathrm{CI} 0.34-1.53, \mathrm{p}=0.40) .{ }^{6}$ The studies by Patchell and Vecht reported longer survival in patients undergoing surgery plus radiotherapy, while the study by Mintz revealed longer survival in patients treated with radiotherapy alone.

\section{Outcome: Lesion recurrence}

The study by Patchel (1B) was the only one to properly describe the results in this outcome. Recurrence in the surgical group totaled $20 \%$ and in the RT group, $52 \%$. The difference was statistically significant $(\mathrm{p}<0.02)$.

\section{Methodological quality analysis}

This trial was classified as Jadad $=3$. The study's strength reached $64.77 \%$.

\section{Evidence summary}

Surgery decreases the recurrence of the lesion as single metastases, compared to radiotherapy.

\section{Outcome: Functional independence}

The three studies examined functional independence.

Patchel and Mitch used the Karnofsky scale. Vecht used the performance status level of the World Health Organization (between 0 and 4).

In the study by Patchel, the surgical group maintained Karnofsky score > 70 in up to 38 weeks of follow-up (on average), and the radiotherapy group maintained this score only up to 8 weeks $(\mathrm{p}<0.0005)$.

In the study by Vecht, there was no difference in functional independence between the groups.

The study by Mitch also revealed no difference in functional independence (Karnofsky) ( $\mathrm{p}=0.98$ ).

\section{Evidence summary}

There is no evidence that surgical treatment preserves the functional independence of patients, compared with radiotherapy.

\section{2) Clinical question}

Is radiosurgery combined with holocranial radiotherapy more effective than radiosurgery or radiotherapy alone ( 1 to 3 metastases)?

\section{Description of the evidence collection method}

The search strategy was conducted in the MedLine (PubMed) database to identify articles published from 1964 to 2013. The objective was to identify studies comparing radiotherapy (combined with radiosurgery) and radiosurgery or radiotherapy alone.

The following terms were searched as "Mesh" and words in the text: "Brain Neoplasms", "Radiosurgery", "Radiotherapy", "Brain Neoplasms/radiotherapy", "Radiotherapy, Adjuvant", "Radiosurgery/methods", "Treatment Outcome", "Radiosurgery/adverse effects".

Related articles were searched from the citations in the primary texts.

- Inclusion criteria: Randomized clinical trials, including those published in English, Spanish and Portuguese.

- Clinical outcomes included were: Functional independence, survival, tumor control, cost-effectiveness, quality of life, cognitive decline and other adverse effects.

\section{Results}

In all, 2,638 articles were identified in the initial search strategy and 29 articles were retrieved based on inclusion criteria. Of these, 19 articles were excluded because they were narrative reviews, evaluation of radiosurgery alone, or treatment of high-grade gliomas.

Another article was manually selected. In the end, nine randomized trials were analyzed to answer this question.

\section{Methodological quality analysis}

Andrews et al. ${ }^{7}$ (1B) (2004) studied, between January 1996 and June 2001, 333 patients in 55 participating centers 167 underwent whole brain radiotherapy and stereotactic radiosurgery, while 164 were allocated for whole brain radiotherapy alone. All patients were aged 18 years or older, and had not been treated with cranial radiotherapy. Entry criteria included contrast-enhanced MRI showing 1-3 brain metastases, with maximum diameter of $4 \mathrm{~cm}$ for the largest lesion and additional lesions not greater than $3 \mathrm{~cm}$ in diameter. Patients with Karnofsky (KPS) score less than 70, hemoglobin concentration below 80 $\mathrm{g} / \mathrm{L}$, absolute neutrophil count below 1,000 or 50,000 cells were excluded. Patients were stratified by number of brain metastases (single metastasis $v$ s. 2-3) and extent of extracranial disease. Randomization was done by blocks using computerized techniques. Sample size was previous- 
ly calculated considering 124 patients per group and eventually adjusted to 326 patients. The analysis of loss to follow-up was done based on intention-to-treat. There is no data on extraction of results.

Kondziolka $^{8}$ (1B) (1999) was a single-center trial discontinued after the interim analysis of 27 patients that revealed significant benefit in the rate of local metastatic control with whole brain radiotherapy plus radiosurgery. This study included patients with 2 to 4 brain metastases sized $25 \mathrm{~mm}$ or less. Local tumor control was also assessed as a primary outcome. No other results were presented. MRI scans were read by an independent observer blinded for treatments.

Aoyama et al. ${ }^{9}$ (1B) (2006) studied patients aged 18 years or older with 1 to 4 brain metastases measuring a maximum of $3 \mathrm{~cm}$ in diameter on MRI divided into two groups: stereotactic radiosurgery alone versus stereotactic radiosurgery combined with whole brain radiotherapy. Eligible patients should have a Karnofsky performance score of 70 or more. The study was conducted at the Hokkaido University (Japan) and ten other centers. Randomization was centralized at the Hokkaido University, in blocks of four. The patients were stratified based on the number of brain metastases (single vs. 2-4), extent of extracranial disease (active $v$ s. stable), and location of the primary tumor (lung $v$ s. other sites).

The study by Chang et al. ${ }^{10}$ (1B) (2009) considered eligible patients who were treated at the Departments of Radiation Oncology and Neurosurgery of MD Anderson Cancer Center, Houston, aged 18 years or older, Karnofsky = 70 or more, with 1-3 brain metastases. Randomization was done by computer (1:1) between the group of stereotactic radiosurgery combined with radiotherapy and stereotactic radiosurgery alone, in blocks of 2, 4, 6, or 8 patients. The sequence was hidden until all interventions were designated. Intention-to-treat analysis was conducted.

Kocher et al. ${ }^{11}$ (1B) studies the role of whole brain radiotherapy after surgery or radiosurgery in a limited number of brain metastases in patients in good conditions with stable systemic cancer. The trial was randomized by center, organized by the European Organisation for Research and Treatment of Cancer. The study was designed to detect a difference of $11 \%$ in the proportion of live patients, with strength of $80 \%$ and $5 \%$ of two-tailed significance. 340 patients were planned to be recruited.

Soffietti R et al. ${ }^{12}$ (1B): This was a multicenter, randomized, international, phase III trial comparing patients undergoing radiosurgery or surgery with whole brain radiotherapy as adjuvant treatment or monitoring.
Results

Outcome: Survival

Andrews et al. ${ }^{7}$ (1B) (2004) studied 333 patients evaluating the effects of the "boost" of radiosurgery in the tumor bed after radiotherapy. There was no benefit in survival between the groups. However, in patients with single metastasis, combined treatment proved to be superior (6.5 months vs. 4.9 months, $\mathrm{p}=0.03$ ) (1B).

Kondziolka et al. ${ }^{8}$ (1B) (1997) studied 27 patients and found no significant differences with respect to survival. The mean survival in the group undergoing combination treatment was 11 months, while the group treated with radiotherapy alone had a survival of 7.5 months $(p=0.22)$. This study was terminated early because an interim analysis showed significant benefit in terms of local tumor control in favor of the combined treatment (evidence level 2B).

Aoyama et al. (2006), in 132 patients, obtained a 7.5 month survival with the combined treatment, and 8 months in the group undergoing radiosurgery alone $(\mathrm{p}=0.42)$ (level of evidence 1B).

Chang et al. (2009) compared the combined treatment with radiosurgery alone in 58 patients. In the radiosurgery group, survival was 15.2 months versus 5.7 in the combined treatment group $(\mathrm{p}=0.003)$ (level of evidence 1B). This study was terminated at 4 months after an interim analysis showed cognitive decline in the combined treatment group.

\section{Evidence summary}

Compared to radiosurgery alone, it is not possible to determine whether the combination treatment is superior or not in terms of survival (A).

\section{Outcome: Functional INDEPENDENCE 3) Clinical question}

Does radiosurgery combined with holocranial radiotherapy increase the time of functional independence compared with radiosurgery or holocranial radiotherapy alone?

\section{Results}

Andrews et al. (2004) found that combination treatment provides less possibility of functional worsening at 6 months $(\mathrm{RR}=0.78$, 95CI $0.61-1, \mathrm{p}=0.05)$ compared with holocranial radiotherapy alone (level of evidence $2 \mathbf{B}$ ).

Kocher et al. (2011) showed that adjuvant radiotherapy does not improve functional outcome of patients undergoing radiosurgery alone. The mean time of worsening of functional status was 10 months (95CI 8.1-11.7) 
for those not treated with radiotherapy vs. 9.5 months for patients undergoing RT (95CI 7.8-11.9), $\mathrm{p}=0.71$ (level of evidence 1B).

Aoyama et al. (1996) did not find benefit in functional prognosis $(\mathrm{p}=0.53)$ or preservation of neurological function $(\mathrm{p}=0.99)$ at 12 months with combined treatment (level of evidence 1B).

\section{Evidence summary}

Radiotherapy combined with holocranial radiotherapy does not increase the time of functional independence compared with radiosurgery (A). However, compared to patients treated with radiotherapy, the combined treatment is superior in terms of functional independence at 6 months (B).

\section{OUTCOME: TUMOR CONTROL 4) Clinical question}

Does radiosurgery combined with holocranial radiotherapy improve tumor control compared with radiosurgery or holocranial radiotherapy alone?

\section{Results}

The study by Kondziolka et al. (1997) ended prematurely after an interim analysis identified considerable benefit in terms of local tumor control with combined treatment compared to radiotherapy alone. The median time to local recurrence was 36 months for the first and 6 months for radiotherapy alone $(\mathrm{p}=0.005)$ (level of evidence $\mathbf{2 B}$ ).

In the study by Kocher et al. (2004), combined treatment led to better local control of tumors at 2 years compared with radiosurgery alone: reduction from $31 \%$ (95CI $22-40 \%$ ) to $19 \%$ (95CI 11-27\%; $\mathrm{p}=0.04$ ). There was also better control of tumors in other locations: a reduction from $48 \%$ (95CI 38-58\%) to 33\% (95CI 24-43\%; $\mathrm{p}=0.023$ ).

In the study by Chang et al. (2009), tumor control at 1 year occurred in $100 \%$ of the patients undergoing combined treatment versus $67 \%$ of tumor control with radiosurgery alone $(\mathrm{p}=0.012)$.

In the study by Aoyama et al. (1996), tumor control at 12 months reached $88.7 \%$ (95CI $80.1-97.3 \%$ ) in the group of combined treatment versus $72.5 \%$ (95CI 60.3$84.7 \%)$ in the group undergoing radiosurgery alone $(\mathrm{p}=0.002)$. Tumor control at 1 year in other sites of the brain was also more favorable than under combined treatment. There was a decline from $73 \%$ to $45 \%(\mathrm{p}=0.02)$.

\section{Evidence summary}

Radiosurgery combined with holocranial radiotherapy reduces the likelihood of local tumor recurrence and the development of new brain lesions (A).

\section{Outcome: Cognitive decline 5) Clinical question}

Is radiosurgery combined with holocranial radiotherapy associated with greater cognitive decline compared with radiosurgery or holocranial radiotherapy alone?

\section{Results}

The study by Chang et al. (2009) had cognitive function as the primary endpoint, which was evaluated by a set of neuropsychological tests covering various domains. This study was terminated early after an interim analysis demonstrated that the possibility of deterioration of learning and memory at 4 months was significantly higher in patients undergoing combined treatment $(52 \%)$ than in patients treated with radiosurgery alone (24\%) (level of evidence 1B).

Aoyama et al. ${ }^{13}$ (2007) used the mini-mental state examination to assess cognitive function, and found that the main factor related to cognitive function is tumor control. Cognitive worsening was earlier the single treatment group ( 7.6 months $v$ s. 16.5 months, $\mathrm{p}=0.05$ ). However, after 36 months, only $14.7 \%$ of patients who underwent combined treatment had no cognitive worsening (level of evidence 2B).

\section{Evidence summary}

Radiosurgery combined with holocranial radiotherapy leads to significant worsening of cognition compared with radiosurgery alone (A).

\section{Outcome: Quality OF LIFE 6) Clinical question}

Is radiosurgery combined with holocranial radiotherapy associated with poorer quality of life compared to radiosurgery or holocranial radiotherapy alone?

\section{Results}

Soffietti et al. (2013) analyzed the aspects of quality of life based on the EORTC QLQ-C30 scale in patients undergoing radiosurgery/surgery alone or combined treatment. Patients who underwent combined treatment had poorer overall quality of life at 9 months $(\mathrm{p}=0.014)(\mathrm{lev}$ el of evidence $\mathbf{2 B}$ ).

\section{Evidence summary}

Radiosurgery combined with holocranial radiotherapy is associated with poorer quality of life (B).

\section{Note}

The different treatment modalities, either combined or alone, produce different effects on various outcomes. The 
recommendations were formulated for each outcome, and the best type of treatment for each type of manifestation should be chosen at the discretion of the attending physician.

\section{RefEREnCES}

1. Patchell RA, Tibbs PA, Walsh JW, Dempsey RJ, Maruyama Y, Kryscio RJ, et al. A randomized trial of surgery in the treatment of single metastases to the brain. N Engl J Med. 1990; 322(8):494-500.

2. Vecht CJ, Haaxma-Reiche H, Noordijk EM, Padberg GW, Voormolen JH, Hoekstra FH, et al. Treatment of single brain metastasis: radiotherapy alone or combined with neurosurgery? Ann Neurol. 1993; 33(6):583-90.

3. Mintz AH, Kestle J, Rathbone MP, Gaspar L, Hugenholtz H, Fisher B, et al. A randomized trial to assess the efficacy of surgery in addition to radiotherapy in patients with a single cerebral metastasis. Cancer. 1996; 78(7):1470-6.

4. Pamar MKB, Torri V, Stewart L. Extracting summary statistics to perform meta-analysis of the published literature for survival endpoints. Stat Med. 1998; 17(24):2815-34

5. Williamson PR, Smith CT, Hutton JL, Marson AG. Aggregate data metaanalysis with time-to-event outcomes. Stat Med. 2002; 21(22):3337-51.

6. Hart MG, Grant R, Walker M, Dickinson H. Surgical resection and whole brain radiation therapy versus whole brain radiation therapy alone for single brain metastases. Cochrane Database Syst Rev. 2005; (1):CD003292.

7. Andrews DW, Scott CB, Sperduto PW, Flanders AE, Gaspar LE, Schell $\mathrm{MC}$, et al. Whole brain radiation therapy with or without stereotactic radiosurgery boost for patients with one to three brain metastases: phase III results of the RTOG 9508 randomised trial. Lancet. 2004; 363(9422):1665-72

8. Kondziolka D, Patel A, Lunsford LD, Kassam A, Flickinger JC. Stereotactic radiosurgery plus whole brain radiotherapy versus radiotherapy alone for patients with multiple brain metastases. Int J Radiat Oncol Biol Phys. 1999; $45(2): 427-34$

9. Aoyama H, Shirato H, Tago M, Nakagawa K, Toyoda T, Hatano K, et al. Stereotactic radiosurgery plus whole-brain radiation therapy vs stereotactic radiosurgery alone for treatment of brain metastases: a randomized controlled trial. JAMA. 2006; 295(21):2483-91.

10. Chang EL, Wefel JS, Hess KR, Allen PK, Lang FF, Kornguth DG, et al. Neurocognition in patients with brain metastases treated with radiosurgery or radiosurgery plus whole-brain irradiation: a randomised controlled trial. Lancet Oncol. 2009; 10(11):1037-44

11. Kocher M, Soffietti R, Abacioglu U, Villà S, Fauchon F, Baumert BG, et al. Adjuvant whole-brain radiotherapy versus observation after radiosurgery or surgical resection of one to three cerebral metastases: results of the EORTC 22952-26001 study. J Clin Oncol. 2011; 29(2):134-41.

12. Soffietti R, Kocher M, Abacioglu UM, Villa S, Fauchon F, Baumert BG, et al. A European Organization for Research and Treatment of Cancer phase III trial of adjuvant whole-brain radiotherapy versus observation in patients with one to three brain metastases from solid tumors after surgical resection or radiosurgery: quality-of-life results. J Clin Oncol. 2013; 31(1):65-72.

13. Aoyama H, Tago M, Kato N, Toyoda T, Kenjyo M, Hirota S, et al. Neurocognitive function of patients with brain metastasis who received either whole brain radiotherapy plus stereotactic radiosurgery or radiosurgery alone. Int J Radiat Oncol Biol Phy.s 2007; 68(5):1388-95. 